\title{
A PRIMITIVE ASSOCIATED TO THE CANTOR- BENDIXSON DERIVATIVE ON THE REAL LINE
}

\section{BORYS ÁLVAREZ-SAMANIEGO and ANDRÉS MERINO}

Núcleo de Investigadores Científicos

Facultad de Ingeniería

Ciencias Físicas y Matemática

Universidad Central del Ecuador (UCE)

Quito

Ecuador

e-mail: balvarez@uce.edu.ec balvarez@impa.br

Facultad de Ciencias

Escuela Politécnica Nacional (EPN)

Quito

Ecuador

e-mail: andres.merino@epn.edu.ec

\begin{abstract}
We consider the class of compact countable subsets of the real numbers $\mathbb{R}$. By using an appropriate partition, up to homeomorphism, of this class we give a detailed proof of a result shown by Mazurkiewicz and Sierpinski related to the cardinality of this partition. Furthermore, for any compact subset of $\mathbb{R}$, we show the existence of a "primitive" related to its Cantor-Bendixson derivative.
\end{abstract}

2010 Mathematics Subject Classification: 54A25, 03E15.

Keywords and phrases: Cantor-Bendixson's derivative, ordinal numbers.

Received July 15, 2016

(ㄷ) 2016 Scientific Advances Publishers 


\section{Introduction}

The earliest ideas of limit point and derived set in the space of the real numbers were both introduced and investigated by Georg Cantor since 1872 (see also [1, 2, 3, 4, 6]) to analyze the convergence set of a trigonometric series. These two concepts have been generalized to the case of any arbitrary topological space. Thus, let $X$ be a topological space and let $A$ be a subset of $X$, we write $A^{\prime}$ to denote the derived set of $A$, that is, the set of all limit points of $A$. The next definition extends the process of taking the derivative of a set for any ordinal number.

Definition 1.1 (Cantor-Bendixson's derivative). Let $A$ be a subset of a topological space. For a given ordinal number $\alpha$, we define, using transfinite recursion, the $\alpha$-th derivative of $A$, written $A^{(\alpha)}$, as follows:

- $A^{(0)}=A$,

- $A^{(\beta+1)}=\left(A^{(\beta)}\right)^{\prime}$, for all ordinal $\beta$,

- $A^{(\lambda)}=\bigcap_{\gamma<\lambda} A^{(\gamma)}$, for all limit ordinal $\lambda \neq 0$.

In this paper, we are initially concerned with the Cantor-Bendixson derivative of compact countable subsets of the real numbers, where a countable set is either a finite set or a countably infinite set. Thus, we consider the set

$$
\mathcal{K}=\{K \subset \mathbb{R}: K \text { is compact and countable }\} .
$$

Moreover, for all $K_{1}, K_{2} \in \mathcal{K}$, we define the relation

$$
K_{1} \sim K_{2} \Leftrightarrow \text { there exists } f: K_{1} \mapsto K_{2} \text { continuous and bijective. }
$$

It is not hard to see that $\sim$ is an equivalence relation on the set $\mathcal{K}$ and since the elements of $\mathcal{K}$ are compact sets, we have that for all $K_{1}, K_{2} \in \mathcal{K}$

$$
K_{1} \sim K_{2} \Leftrightarrow \text { there exists } f: K_{1} \mapsto K_{2} \text { homeomorphism. }
$$


Therefore, there is a partition of the set $\mathcal{K}$, and we denote by

$$
\mathscr{K}=\mathcal{K} / \sim
$$

the set of all equivalence classes of $\mathcal{K}$.

In 1920, Mazurkiewicz and Sierpinski [7] showed that the cardinality of $\mathscr{K}$ is $\aleph_{1}$. In Section 2 , we show in detail that for any countable ordinal number $\alpha$, and for any $p \in \omega$, there is a set $K \in \mathcal{K}$ such that $K^{(\alpha)}$ has exactly $p$ elements. This last fact was first briefly mentioned by Cantor in [3]. The results shown in Section 2 allow us to prove, in Theorem 3.4 , that the cardinality of $\mathscr{K}$ is greater than or equal to $\aleph_{1}$. On the other hand, the cardinality of $\mathscr{K}$ is smaller than or equal to $\aleph_{1}$ as a consequence of Theorem 3.3.

Section 3 considers Cantor-Bendixson's characteristic, denoted by $\mathcal{C B}$. First, we show that for any element $K \in \mathcal{K}$ with $\mathcal{C B}(K)=(\alpha, p)$, we get $p=0$ if and only if $K=\varnothing$. Moreover, we use Lemma 3.6 to prove Theorem 3.3, where the injectivity of function $\widetilde{\mathcal{C B}}$, defined in (3.12), is shown. These two last results were first mentioned in [7]; however, for the sake of completeness, we include here their detailed proofs. Finally, Theorem 3.5 shows that for any compact subset of the reals, there exists a primitive-like set connected with its Cantor-Bendixson derivative.

We recall that if $F$ is a closed subset of $\mathbb{R}$, then $\left(F^{(\alpha)}\right)_{\alpha \in \mathbf{O R}}$ is a decreasing family of closed subsets of the real line. Furthermore, if $K \in \mathcal{K}$, then $\left(K^{(\alpha)}\right)_{\alpha \in \mathbf{O R}}$ is a decreasing family of elements of $\mathcal{K}$.

We denote by OR, the class of all ordinal numbers. Moreover, $\omega$ is used to designate the set of all natural numbers and $\Omega$ represents the set of all countable ordinal numbers. In addition, the cardinality of a set $B$ is denoted by $|B|$. 


\section{A Family of Elements in $\mathcal{K}$ Having a Cantor-Bendixson's Derivative with any Given Finite Number of Elements}

First, we remark that any finite subset of $\mathbb{R}$ is an element of $\mathcal{K}$ with empty derived set. Thus, a set of this kind satisfies the property that its Cantor-Bendixson's derivative is empty for all ordinal number greater than or equal to 1 . The following theorem let us find some elements belonging to $\mathcal{K}$ not satisfying this last property. The main idea of the next result was given in [3], for completeness, we present below its proof in detail.

Theorem 2.1. For any countable ordinal number $\alpha \in \Omega$, and for all $a, b \in \mathbb{R}$ such that $a<b$, there is a set $K \in \mathcal{K}$ such that $K \subset(a, b]$ and $K^{(\alpha)}=\{b\}$.

Proof. We will use transfinite induction.

(a) First, we consider the case $\alpha=0$. For any $a, b \in \mathbb{R}$ such that $a<b$, the result follows by taking the set $K=\{b\} \in \mathcal{K}$.

(b) Now, we suppose that for a given countable ordinal number $\alpha \in \Omega$, and for all $c, d \in \mathbb{R}$ such that $c<d$, there is a set $\widetilde{K} \in \mathcal{K}$ such that $\tilde{K} \subset(c, d]$ and $\widetilde{K}^{(\alpha)}=\{d\}$ Let $a, b \in \mathbb{R}$ be such that $a<b$. We take a strictly increasing sequence, $\left(x_{n}\right)_{n \in \omega}$, in $(a, b]$ such that $x_{n} \rightarrow b$ as $n \rightarrow+\infty$. Defining $x_{-1}:=a$ and applying the hypothesis to the real numbers $x_{m-1}<x_{m}, m \in \omega$, it follows that there exists a sequence of sets $\left(K_{m}\right)_{m \in \omega}$ such that for all $m \in \omega, K_{m} \in \mathcal{K}, K_{m} \subset\left(x_{m-1}, x_{m}\right]$ and $K_{m}^{(\alpha)}=\left\{x_{m}\right\}$. Now, we define the set

$$
K:=\biguplus_{m \in \omega} K_{m} \biguplus\{b\} .
$$


The set $K$, given in (2.1), satisfies the following properties:

- $K \subset(a, b]$, since $K_{m} \subset\left(x_{m-1}, x_{m}\right] \subset(a, b]$, for all $m \in \omega$.

- $K$ is countable, since it is the countable union of countable sets.

- $K$ is compact. In fact, given $\left(A_{i}\right)_{i \in I}$ an open cover of $K$, there is a $j \in I$ such that $b \in A_{j}$. Since $A_{j}$ is an open set and $\left(x_{n}\right)_{n \in \omega}$ is a strictly increasing sequence that converges to $b$, there exists $N_{1} \in \omega$ such that $K_{n} \subset A_{j}$ for all $n \in \omega$ with $n>N_{1}$. On the other hand, the set $C:=\biguplus_{n=0}^{N_{1}} K_{n}$ is compact, since it is the finite union of compact sets. Thus, $C$ has a finite open subcover $\left(A_{i}\right)_{i \in J}$. Then, $\left(A_{i}\right)_{i \in J \cup\{j\}}$ is a finite open subcover of $K$.

- For all ordinal number $\beta$ with $\beta \leq \alpha$,

$$
K^{(\beta)}=\biguplus_{m \in \omega} K_{m}^{(\beta)} \biguplus\{b\} .
$$

Last expression is obtained by using transfinite induction on $\beta$. In fact, the case $\beta=0$ is immediate from (2.1). Now, we suppose that for a given ordinal number $\beta<\alpha$, (2.2) holds. Since $\beta+1 \leq \alpha$, we have that $K_{m}^{(\alpha)} \subset K^{(\alpha)} \subset K^{(\beta+1)}$, for all $m \in \omega$. Moreover, since $x_{m} \in K_{m}^{(\alpha)} \subset K^{(\beta+1)}$, for all $m \in \omega$, and $x_{m} \rightarrow b$ as $m \rightarrow+\infty$, we see that $b \in K^{(\beta+1)}$. Therefore,

$$
\biguplus_{m \in \omega} K_{m}^{(\beta+1)} \biguplus\{b\} \subset K^{(\beta+1)} .
$$

In order to prove the other inclusion, let $x \in K^{(\beta+1)}$. Using the induction hypothesis, we see that

$$
K^{(\beta+1)} \subset K^{(\beta)}=\biguplus_{m \in \omega} K_{m}^{(\beta)} \biguplus\{b\} .
$$

Therefore, either $x=b$ or $x \in K_{m}^{(\beta)}$ for some $m \in \omega$. If $x=b$, then there is nothing else to prove. If $x \neq b$, there exists $M \in \omega$ such that 


$$
x \in K_{M}^{(\beta)} \subset K_{M} \subset\left(x_{M-1}, x_{M}\right] .
$$

We claim that $x \in K_{M}^{(\beta+1)}$. To prove the last assertion, we suppose, by contradiction, that $x \notin K_{M}^{(\beta+1)}$. Thus, $x$ is an isolated point of $K_{M}^{(\beta)}$. However, we know that $\left\{x_{M}\right\}=K_{M}^{(\alpha)} \subset K_{M}^{(\beta+1)}$. Then, $x \neq x_{M}$. Thus, there exists $\epsilon>0$ such that $(x-\varepsilon, x+\varepsilon) \subset\left(x_{M-1}, x_{M}\right)$ and

$$
(x-\varepsilon, x+\varepsilon) \cap K_{M}^{(\beta)}=\{x\} .
$$

Moreover, since $(x-\varepsilon, x+\varepsilon) \subset\left(x_{M-1}, x_{M}\right)$, we conclude that for all $m \in \omega \backslash\{M\}$,

$$
(x-\varepsilon, x+\varepsilon) \cap K_{m}^{(\beta)}=\varnothing .
$$

Hence,

$$
\begin{aligned}
\{x\} & =(x-\varepsilon, x+\varepsilon) \cap\left(\biguplus_{m \in \omega} K_{m}^{(\beta)} \biguplus\{b\}\right) \\
& =(x-\varepsilon, x+\varepsilon) \cap K^{(\beta)},
\end{aligned}
$$

where in the last equality we have used the assumption that (2.2) holds for $\beta$. Even so, this last expression is a contradiction with the fact that $x \in K^{(\beta+1)}$. Then, $x \in K_{M}^{(\beta+1)}$. Thus,

$$
K^{(\beta+1)} \subset \biguplus_{m \in \omega} K_{m}^{(\beta+1)} \biguplus\{b\} .
$$

Using (2.3) and (2.4), we get

$$
K^{(\beta+1)}=\biguplus_{m \in \omega} K_{m}^{(\beta+1)} \biguplus\{b\} .
$$

Finally, let $\gamma \neq 0$ be a limit ordinal such that $\gamma \leq \alpha$ and suppose that

$$
K^{(\delta)}=\biguplus_{m \in \omega} K_{m}^{(\delta)} \biguplus\{b\},
$$


for all ordinal number $\delta$ such that $\delta<\gamma$. Following a similar procedure to the one performed above to obtain (2.3), we have that

$$
\biguplus_{m \in \omega} K_{m}^{(\gamma)} \biguplus\{b\} \subset K^{(\gamma)} .
$$

To obtain the other inclusion, let $x \in K^{(\gamma)}$. Using the induction hypothesis (2.5), we see that

$$
K^{(\gamma)}:=\bigcap_{\delta<\gamma} K^{(\delta)}=\bigcap_{\delta<\gamma}\left(\biguplus_{m \in \omega} K_{m}^{(\delta)} \biguplus\{b\}\right) .
$$

Then, either $x=b$ or for all ordinal number $\delta$ such that $\delta<\gamma$, there exists $m \in \omega$ such that $x \in K_{m}^{(\delta)}$. If $x=b$, then there is nothing left to prove. If $x \neq b$, there exists $M \in \omega$ such that $x \in K_{M}^{(0)}=K_{M} \subset\left(x_{M-1}, x_{M}\right]$. We claim now that for all ordinal number $\delta$ such that $\delta<\gamma, x \in K_{M}^{(\delta)}$. In fact, we suppose, by contradiction, that there is an ordinal number $\delta_{0}$ with $\delta_{0}<\gamma$ and such that $x \notin K_{M}^{\left(\delta_{0}\right)}$. However, we know that there exists $m_{0} \in \omega$ with $m_{0} \neq M$ such that $x \in K_{m_{0}}^{\left(\delta_{0}\right)} \subset K_{m_{0}} \subset\left(x_{m_{0}-1}, x_{m_{0}}\right]$. Since $m_{0} \neq M$, we get $\left(x_{m_{0}-1}, x_{m_{0}}\right] \cap\left(x_{M-1}, x_{M}\right]=\varnothing$, which is a contradiction with the fact that $x \in\left(x_{m_{0}-1}, x_{m_{0}}\right] \cap\left(x_{M-1}, x_{M}\right]$. Therefore,

$$
x \in \bigcap_{\delta<\gamma} K_{M}^{(\delta)}=: K_{M}^{(\gamma)} \subset \biguplus_{m \in \omega} K_{m}^{(\gamma)} .
$$

Then,

$$
K^{(\gamma)} \subset \biguplus_{m \in \omega} K_{m}^{(\gamma)} \biguplus\{b\}
$$

By (2.6) and (2.7), we have that

$$
K^{(\gamma)}=\biguplus_{m \in \omega} K_{m}^{(\gamma)} \biguplus\{b\} .
$$

Hence, (2.2) holds for all ordinal number $\beta$ such that $\beta \leq \alpha$. 
Applying now (2.2) to the ordinal number $\alpha$, and since $K_{m}^{(\alpha)}=\left\{x_{m}\right\}$, for all $m \in \omega$, we conclude that

$$
\begin{aligned}
K^{(\alpha)} & =\biguplus_{m \in \omega} K_{m}^{(\alpha)} \biguplus\{b\} \\
& =\biguplus_{m \in \omega}\left\{x_{m}\right\} \biguplus\{b\} \\
& =\left\{x_{m}: m \in \omega\right\} \biguplus\{b\} .
\end{aligned}
$$

Therefore,

$$
K^{(\alpha+1)}=\left(K^{(\alpha)}\right)^{\prime}=\{b\} .
$$

(c) Finally, let $\lambda \neq 0$ be a countable limit ordinal number. We suppose that for all ordinal number $\rho$ such that $\rho<\lambda$ and for all $c, d \in \mathbb{R}$ such that $c<d$, there is a set $\widetilde{K} \in \mathcal{K}$ such that $\tilde{K} \subset(c, d]$ and $\widetilde{K}^{(\rho)}=\{d\}$. Since $\lambda$ is a countable limit ordinal number, there exits a strictly increasing sequence $\left(\rho_{n}\right)_{n \in \omega}$ in $\Omega$ such that $\rho_{n}<\lambda$, for all $n \in \omega$, and $\sup \left\{\rho_{n}: n \in \omega\right\}=\lambda$. Let $a, b \in \mathbb{R}$ be such that $a<b$. We take a strictly increasing sequence, $\left(x_{n}\right)_{n \in \omega}$, in $(a, b]$ such that $x_{n} \rightarrow b$ as $n \rightarrow+\infty$. Defining again $x_{-1}=a$ and applying the hypothesis to the real numbers $x_{m-1}<x_{m}$, and the ordinal number $\rho_{m}, m \in \omega$, it follows that there exists a sequence of sets $\left(K_{m}\right)_{m \in \omega}$ such that for all $m \in \omega, K_{m} \in \mathcal{K}, K_{m} \subset\left(x_{m-1}, x_{m}\right]$ and $K_{m}^{\left(\rho_{m}\right)}=\left\{x_{m}\right\}$. We also define, as in the previous case, the set

$$
K:=\biguplus_{m \in \omega} K_{m} \biguplus\{b\} .
$$

It can be shown, similarly to the case (b) above, that the set $K$, defined in (2.8), satisfies the following properties:

- $K \subset(a, b]$.

- $K$ is countable. 
- $K$ is compact.

- For all ordinal number $\rho$ with $\rho \leq \lambda$,

$$
K^{(\rho)}=\biguplus_{m \in \omega} K_{m}^{(\rho)} \biguplus\{b\} .
$$

Last expression is obtained by using transfinite induction on $\rho$. In fact, the case $\rho=0$ is immediate from (2.8). Now, we suppose that for a given ordinal number $\rho<\lambda$, (2.9) holds. Since $\lambda$ is a limit ordinal, we have that $\rho+1<\lambda$, and then there exists $N \in \omega$ such that $\rho+1<\rho_{m}$

for all $m \in \omega$ with $m>N$. Therefore, $x_{m} \in K_{m}^{\left(\rho_{m}\right)} \subset K_{m}^{(\rho+1)} \subset K^{(\rho+1)}$, for all $m \in \omega$ with $m>N$, and since $x_{m} \rightarrow b$ as $m \rightarrow+\infty$, we see that $b \in K^{(\rho+1)}$. Then,

$$
\biguplus_{m \in \omega} K_{m}^{(\rho+1)} \biguplus\{b\} \subset K^{(\rho+1)} .
$$

In order to prove the other inclusion, let $x \in K^{(\rho+1)}$. Using the induction hypothesis, we see that

$$
K^{(\rho+1)} \subset K^{(\rho)}=\biguplus_{m \in \omega} K_{m}^{(\rho)} \biguplus\{b\} .
$$

Therefore, either $x=b$ or $x \in K_{m}^{(p)}$ for some $m \in \omega$. If $x=b$, then there is nothing else to prove. If $x \neq b$, there exists $M \in \omega$ such that

$$
x \in K_{M}^{(\rho)} \subset K_{M} \subset\left(x_{M-1}, x_{M}\right] .
$$

Since $K_{M}^{\left(\rho_{M}+1\right)}=\varnothing$, we have that $\rho<\rho_{M}+1$, that is $\rho \leq \rho_{M}$. We claim that $x \in K_{M}^{(\rho+1)}$. To prove the last assertion, we suppose, by contradiction, that $x \notin K_{M}^{(\rho+1)}$. Thus, $x$ is an isolated point of $K_{M}^{(\rho)}$. However, we know that $K_{M} \cap K_{M+1}=\varnothing$, then $x \notin K_{M+1}$. Hence, $x \notin K_{M+1}^{(\rho)}$. Thus, there exists $\varepsilon>0$ such that $(x-\varepsilon, x+\varepsilon) \subset\left(x_{M-1}, x_{M+1}\right)$, $(x-\varepsilon, x+\varepsilon) \cap K_{M+1}^{(\rho)}=\varnothing$, and 


$$
(x-\varepsilon, x+\varepsilon) \cap K_{M}^{(\rho)}=\{x\},
$$

where in the second expression above we have used the fact that $K_{M+1}^{(\rho)}$ is a closed subset of $\mathbb{R}$. Moreover, since $(x-\varepsilon, x+\varepsilon) \subset\left(x_{M-1}, x_{M+1}\right)$, we conclude that for all $m \in \omega \backslash\{M\}$,

$$
(x-\varepsilon, x+\varepsilon) \cap K_{m}^{(\rho)}=\varnothing .
$$

Hence,

$$
\begin{aligned}
\{x\} & =(x-\varepsilon, x+\varepsilon) \cap\left(\biguplus_{m \in \omega} K_{m}^{(\rho)} \biguplus\{b\}\right) \\
& =(x-\varepsilon, x+\varepsilon) \cap K^{(\rho)},
\end{aligned}
$$

where in the last equality we have used the assumption that (2.9) holds for $\rho$. Nevertheless, this last expression is a contradiction with the fact that $x \in K^{(\rho+1)}$. Then, $x \in K_{M}^{(\rho+1)}$. Thus,

$$
K^{(\rho+1)} \subset \biguplus_{m \in \omega} K_{m}^{(\rho+1)} \biguplus\{b\} .
$$

Using (2.10) and (2.11), we get

$$
K^{(\rho+1)}=\biguplus_{m \in \omega} K_{m}^{(\rho+1)} \biguplus\{b\} .
$$

Finally, let $\gamma \neq 0$ be a limit ordinal such that $\gamma \leq \lambda$ and suppose that

$$
K^{(\delta)}=\biguplus_{m \in \omega} K_{m}^{(\delta)} \biguplus\{b\},
$$

for all ordinal number $\delta$ such that $\delta<\gamma$. We have, using (2.12), that

$$
\begin{aligned}
\biguplus_{m \in \omega} K_{m}^{(\gamma)} \biguplus\{b\} & =\biguplus_{m \in \omega}\left(\bigcap_{\delta<\gamma} K_{m}^{(\delta)}\right) \biguplus\{b\} \\
& \subset \bigcap_{\delta<\gamma}\left(\biguplus_{m \in \omega} K_{m}^{(\delta)}\right) \biguplus\{b\}
\end{aligned}
$$




$$
\begin{aligned}
& =\bigcap_{\delta<\gamma}\left(\biguplus_{m \in \omega} K_{m}^{(\delta)} \biguplus\{b\}\right) \\
& =\bigcap_{\delta<\gamma} K^{(\delta)} \\
& =K^{(\gamma)} .
\end{aligned}
$$

To get the other inclusion, we can follow a similar procedure to the one performed above to obtain (2.7). Thus, we have that

$$
K^{(\gamma)} \subset \biguplus_{m \in \omega} K_{m}^{(\gamma)} \biguplus\{b\} .
$$

By (2.13) and (2.14), we obtain

$$
K^{(\gamma)}=\biguplus_{m \in \omega} K_{m}^{(\gamma)} \biguplus\{b\} .
$$

Consequently, (2.9) holds for all ordinal number $\rho$ such that $\rho \leq \lambda$. Furthermore, since for all $m \in \omega, \rho_{m}+1<\lambda$, it follows that for all $m \in \omega$

$$
K_{m}^{(\lambda)} \subset K_{m}^{\left(\rho_{m}+1\right)}=\left(K_{m}^{\left(\rho_{m}\right)}\right)^{\prime}=\left(\left\{x_{m}\right\}\right)^{\prime}=\varnothing .
$$

Therefore,

$$
K^{(\lambda)}=\biguplus_{m \in \omega} K_{m}^{(\lambda)} \biguplus\{b\}=\{b\} .
$$

From (a), (b), and (c), the theorem is proved.

The next lemma will be used in the proof of Corollary 2.1 below.

Lemma 2.1. Suppose that $n \in \omega$. Let $F_{1}, F_{2}, \ldots, F_{n}$ be closed subsets of $\mathbb{R}$. Then, for all ordinal number $\alpha \in \mathrm{OR}$, we have that

$$
\left(\bigcup_{k=1}^{n} F_{k}\right)^{(\alpha)}=\bigcup_{k=1}^{n} F_{k}^{(\alpha)}
$$


Proof. The general case, $n \in \omega$, is a consequence of the result for $n=2$ and the principle of finite induction. Thus, we suppose that $n=2$. We will now use transfinite induction.

(a) If $\alpha=0$, then there is nothing else to prove.

(b) We now suppose that for a given ordinal number $\alpha \in \mathrm{OR}$, $\left(F_{1} \cup F_{2}\right)^{(\alpha)}=F_{1}^{(\alpha)} \cup F_{2}^{(\alpha)}$. Therefore,

$$
\left(F_{1} \cup F_{2}\right)^{(\alpha+1)}=\left(\left(F_{1} \cup F_{2}\right)^{(\alpha)}\right)^{\prime}=\left(F_{1}^{(\alpha)} \cup F_{2}^{(\alpha)}\right)^{\prime}=F_{1}^{(\alpha+1)} \cup F_{2}^{(\alpha+1)},
$$

where in the last equation we have used the fact that the derived set of a finite union of subsets of a metric space equals the union of their derived sets.

(c) Finally, let $\lambda \neq 0$ be a limit ordinal number. We suppose that for all $\beta \in \mathrm{OR}$ such that $\beta<\lambda,\left(F_{1} \cup F_{2}\right)^{(\beta)}=F_{1}^{(\beta)} \cup F_{2}^{(\beta)}$. Then,

$$
\begin{aligned}
F_{1}^{(\lambda)} \cup F_{2}^{(\lambda)} & =\bigcap_{\beta<\lambda} F_{1}^{(\beta)} \cup \bigcap_{\beta<\lambda} F_{2}^{(\beta)} \\
& \subset \bigcap_{\beta<\lambda}\left(F_{1}^{(\beta)} \cup F_{2}^{(\beta)}\right) \\
& =\bigcap_{\beta<\lambda}\left(F_{1} \cup F_{2}\right)^{(\beta)} \\
& =\left(F_{1} \cup F_{2}\right)^{(\lambda)} .
\end{aligned}
$$

In order to prove the other inclusion, we take $x \in\left(F_{1} \cup F_{2}\right)^{(\lambda)}$. We suppose, for the sake of contradiction, that $x \notin F_{1}^{(\lambda)}$ and $x \notin F_{2}^{(\lambda)}$. Thus, there exist $\beta_{1}, \beta_{2} \in \mathrm{OR}$, with $\beta_{1}<\lambda$ and $\beta_{2}<\lambda$, such that $x \notin F_{1}^{\left(\beta_{1}\right)}$ and $x \notin F_{2}^{\left(\beta_{2}\right)}$. If $\beta_{1} \leq \beta_{2}$, then $F_{1}^{\left(\beta_{2}\right)} \subset F_{1}^{\left(\beta_{1}\right)}$. Hence, $x \notin F_{1}^{\left(\beta_{2}\right)} \cup F_{2}^{\left(\beta_{2}\right)}$ 
$=\left(F_{1} \cup F_{2}\right)^{\left(\beta_{2}\right)}$, which contradicts the fact that $x \in\left(F_{1} \cup F_{2}\right)^{(\lambda)}=$ $\bigcap_{\beta<\lambda}\left(F_{1} \cup F_{2}\right)^{(\beta)}$. The proof of the other case, $\beta_{2}<\beta_{1}$, is similar.

Therefore,

$$
\left(F_{1} \cup F_{2}\right)^{(\lambda)}=F_{1}^{(\lambda)} \cup F_{2}^{(\lambda)}
$$

Consequently, the lemma is proved.

The following result is a generalization of Theorem 2.1.

Corollary 2.1. Given any countable ordinal number $\alpha$ and given any $p \in \omega$, there exists $K \in \mathcal{K}$ such that $\left|K^{(\alpha)}\right|=p$.

Proof. Let $\alpha \in \Omega$. If $p=0$, we take $K=\varnothing$. If $p \in \omega \backslash\{0\}$, it is enough to apply Theorem 2.1 to a collection of $p$ pairwise disjoint intervals. Thus, for all $k \in\{1, \ldots, p\}$, there exists $K_{k} \in \mathcal{K}$, such that $K_{k}^{(\alpha)}$ has only one element, and $K_{i} \cap K_{j}=\varnothing$ for $i, j \in\{1, \ldots, p\}$ with $i \neq j$. We now define

$$
K:=\biguplus_{k=1}^{p} K_{k}
$$

Hence, $K \in \mathcal{K}$ and, using Lemma 2.1, we get

$$
K^{(\alpha)}=\biguplus_{k=1}^{p} K_{k}^{(\alpha)}
$$

Therefore, $K^{(\alpha)}$ has exactly $p$ elements.

Remark 2.1. Even though the proofs of (2.2) and (2.9) are similar, it is worth mentioning that they are not identical. In fact, to prove (2.2) we have that $\alpha \in \Omega$ and for all $m \in \omega, K_{m}^{(\alpha)}=\left\{x_{m}\right\}$. On the other hand, to obtain (2.9), we consider $\lambda \neq 0$ a countable limit ordinal and a strictly increasing sequence $\left(\rho_{m}\right)_{m \in \omega}$ in $\Omega$, with $\sup \left\{\rho_{m}: m \in \omega\right\}=\lambda$, such that for all $m \in \omega, \rho_{m}<\lambda$ and $K_{m}^{\left(\rho_{m}\right)}=\left\{x_{m}\right\}$, where $\rho_{m}$ depends on $m$. 
In addition, we point out that the process developed to obtain (2.13) can also be used to get (2.3), (2.6), and (2.10).

\section{Some Results Concerning Cantor-Bendixson's Derivative}

It is a well-known fact that, for all $K \in \mathcal{K},\left(K^{(\alpha)}\right)_{\alpha \in \mathrm{OR}}$ is a decreasing family of elements of $\mathcal{K}$. The following two results were first proved by Cantor in [5] and they imply that for all $K \in \mathcal{K},\left(K^{(\alpha)}\right)_{\alpha \in \mathrm{OR}}$ is in fact a strictly decreasing family of sets in $\mathcal{K}$ up to a countable ordinal number and such that all of its subsequent derivative sets are empty.

Lemma 3.1. If $K \in \mathcal{K}$ and $K \neq \varnothing$, then $K^{\prime} \neq K$.

The above lemma implies the following theorem.

Theorem 3.1. If $K \in \mathcal{K}$, then there exists a countable ordinal number $\beta$ such that $K^{(\beta)}$ is finite.

Since $\Omega$ is a well-ordered set, by the previous theorem, we see that for all $K \in \mathcal{K}$, there exists the smallest countable ordinal number $\alpha$ such that $K^{(\alpha)}$ is finite. We can now give the next definition.

Definition 3.1 (Cantor-Bendixson's characteristic). Let $K \in \mathcal{K}$. We say that $(\alpha, p) \in \Omega \times \omega$ is the Cantor-Bendixson characteristic of $K$ if $\alpha$ is the smallest countable ordinal number such that $K^{(\alpha)}$ is finite and $\left|K^{(\alpha)}\right|=p$. In this case, we write $\mathcal{C B}(K)=(\alpha, p)$.

By Theorem 2.1, for all countable ordinal number $\alpha$, there exists a set $K \in \mathcal{K}$ having Cantor-Bendixson's characteristic $(\alpha, 1)$. Furthermore, by Corollary 2.1, we have that for all $p \in \omega \backslash\{0\}$ and for all $\alpha \in \Omega$, there exists $K \in \mathcal{K}$ such that $\mathcal{C B}(K)=(\alpha, p)$. In addition, we obviously see that $\mathcal{C B}(\varnothing)=(0,0)$. Moreover, we have the next result concerning the empty set. 
Proposition 3.1. Let $K \in \mathcal{K}$ be such that $\mathcal{C B}(K)=(\alpha, p) \in \Omega \times \omega$. Then, $p=0$ if and only if $K=\varnothing$.

Proof. If $K=\varnothing$, then $\mathcal{C B}(K)=(0,0)$, and thus the result holds. Now, we suppose that $K \neq \varnothing$. We consider three cases.

- If $\alpha=0$, then $K=K^{(0)}$ is finite. Since $K \neq \varnothing$, we have that $\left|K^{(0)}\right| \neq 0$. Hence, $p \neq 0$.

- We suppose now that $\alpha$ is a nonzero limit ordinal. Then, for all $\beta \in \Omega$ such that $\beta<\alpha, K^{(\beta)}$ is infinite. Therefore, $\left(K^{(\beta)}\right)_{\beta<\alpha}$ is a decreasing nested family of nonempty compact subsets of $\mathbb{R}$. By using the Cantor intersection theorem, we obtain

$$
K^{(\alpha)}=\bigcap_{\beta<\alpha} K^{(\beta)} \neq \varnothing .
$$

Then, $\left|K^{(\alpha)}\right| \neq 0$, and so $p \neq 0$.

- Finally, we assume that $\alpha$ is a successor ordinal. Thus, there exists an ordinal $\beta \in \Omega$ such that $\beta+1=\alpha$. Since $\beta<\alpha$, it follows that $K^{(\beta)}$ is infinite. Then,

$$
K^{(\alpha)}=K^{(\beta+1)}=\left(K^{(\beta)}\right)^{\prime} \neq \varnothing
$$

Therefore, $\left|K^{(\alpha)}\right| \neq 0$. Hence, $p \neq 0$.

\subsection{Partition of $\mathcal{K}$}

In this subsection, we show some general results concerning the equivalence relation $\sim$ defined on the set $\mathcal{K}$ by (1.2).

Proposition 3.2. Let $K_{1}, K_{2} \in \mathcal{K}$ be such that $K_{1} \sim K_{2}$. Then, $K_{1}^{\prime} \sim K_{2}^{\prime}$. More precisely, if $f$ is a homeomorphism of $K_{1}$ onto $K_{2}$, then $\left.f\right|_{K_{1}^{\prime}}$ is also a homeomorphism of $K_{1}^{\prime}$ onto $K_{2}^{\prime}$. 
Proof. Since the image of a limit point, under a homeomorphism, is also a limit point, we see that $f\left(K_{1}^{\prime}\right)=K_{2}^{\prime}$. Hence, $\left.f\right|_{K_{1}^{\prime}}: K_{1}^{\prime} \mapsto K_{2}^{\prime}$ is a homeomorphism. Therefore, $K_{1}^{\prime} \sim K_{2}^{\prime}$.

By using transfinite induction, we get the following result:

Corollary 3.1. Let $K_{1}, K_{2} \in \mathcal{K}$ be such that $K_{1} \sim K_{2}$, and let $\alpha$ be any ordinal number. Then, $K_{1}^{(\alpha)} \sim K_{2}^{(\alpha)}$. More precisely, if $f$ is a homeomorphism of $K_{1}$ onto $K_{2}$, then $\left.f\right|_{K_{1}^{(\alpha)}}$ is also a homeomorphism of $K_{1}^{(\alpha)}$ onto $K_{2}^{(\alpha)}$.

It follows from the last corollary that if $K_{1}, K_{2} \in \mathcal{K}, K_{1} \sim K_{2}$ and $\mathcal{C B}\left(K_{1}\right)=(\alpha, p) \in \Omega \times \omega$, then there exists a bijective function of $K_{1}^{(\alpha)}$ onto $K_{2}^{(\alpha)}$. Therefore, $\left|K_{2}^{(\alpha)}\right|=\left|K_{1}^{(\alpha)}\right|=p$. Hence, $\mathcal{C B}\left(K_{2}\right)=(\alpha, p)$. This last result about the Cantor-Bendixson characteristic, which was given by Mazurkiewicz and Sierpinski in [7], is expressed in the following theorem:

Theorem 3.2. If $K_{1}, K_{2} \in \mathcal{K}$ and $K_{1} \sim K_{2}$, then $\mathcal{C B}\left(K_{1}\right)=\mathcal{C B}\left(K_{2}\right)$.

The above theorem shows that the Cantor-Bendixson characteristic is preserved for equivalent elements of $\mathcal{K}$, i.e., given $K \in \mathcal{K}$, we have that $\mathcal{C B}\left(K_{1}\right)=\mathcal{C B}(K)$, for all $K_{1} \in[K]$, where $[K]$ denotes the equivalence class of $K$. The reciprocal of Theorem 3.2, which was likewise given by Mazurkiewicz and Sierpinski in [7], is also true, and for completeness we give a more explicit proof of this fact in Theorem 3.3 below. In the following, we consider any ordinal number as a topological space with the order topology. Lemmas 3.2 to 3.6 will be used in the proof of Theorem 3.3 .

Lemma 3.2. Let $K \in \mathcal{K}$ be such that $\mathcal{C B}(K)=(1,1)$. Then, there exists a homeomorphism of K onto $\omega+1$. 
Proof. There is an $x \in \mathbb{R}$ such that $K^{\prime}=\{x\}$. The set $K \backslash K^{\prime}$ is infinite and countable. Therefore, there exists a bijective function $g$ of $K \backslash K^{\prime}$ onto $\omega$. Now, we define

$$
\begin{aligned}
f: K & \mapsto \omega+1 \\
z & \mapsto f(z)= \begin{cases}g(z), & \text { if } z \neq x, \\
\omega, & \text { if } z=x .\end{cases}
\end{aligned}
$$

We see that $f$ is a bijective function. Furthermore, since $\omega+1$ is a compact topological space, $(\omega+1)^{\prime}=\{\omega\}, f$ is an injective function, and $f\left(K^{\prime}\right)=f(\{x\})=\{\omega\}$, we have that $f$ is a continuous function. Moreover, since $\omega+1$ is a Hausdorff space, it follows that $f$ is in fact a homeomorphism.

Lemma 3.3. Let $\alpha$ be a countable ordinal number such that $\alpha>1$. Suppose that for all ordinal number $\beta$ such that $0<\beta<\alpha$ and for all $\tilde{K} \in \mathcal{K}$ such that $\mathcal{C B}(\tilde{K})=(\beta, p) \in \Omega \times(\omega \backslash\{0\})$, there exists a homeomorphism $\tilde{f}$ of $\widetilde{K}$ onto $\omega^{\beta} \cdot p+1$. Then, for all $K \in \mathcal{K}$ such that $\mathcal{C B}(K)=(\alpha, 1)$, there exists a homeomorphism of $K$ onto $\omega^{\alpha}+1$.

Proof. Let $K \in \mathcal{K}$ be such that $\mathcal{C B}(K)=(\alpha, 1)$. Then, there exists an $x \in K$ such that $K^{(\alpha)}=\{x\}$. We have that $x \in K^{(\alpha)} \subset K^{\prime \prime}$. Thus, $x$ is a limit point of $K^{\prime}$. Hence, there exists a strictly increasing or strictly decreasing sequence $\left(x_{n}\right)_{n \in \omega}$ in $K^{\prime}$ such that it converges to $x$. We suppose that $\left(x_{n}\right)_{n \in \omega}$ is an strictly increasing sequence in $K^{\prime}$, the other case is similar.

We claim that for all $n \in \omega$, we can take $r_{n}>0$ such that $x_{n}<x-$ $r_{n}<x_{n+1}$ and $x-r_{n}, x+r_{n} \notin K$. In fact, if we suppose the contrary, then there exists $l \in \omega$ such that

$$
\left[x_{l}-x, x_{l+1}-x\right] \subset\{r \in \mathbb{R}: x-r \in K \text { or } x+r \in K\} .
$$


However, the set on the right-hand side of the last inclusion is countable, which is a contradiction. Hence, the claim is proved. We remark that the sequence $\left(r_{n}\right)_{n \in \omega}$ converges to 0 as $n$ goes to infinity. We now define the sets

$$
\begin{aligned}
& K_{0}=K \cap\left(\left(-\infty, x-r_{0}\right] \cup\left[x+r_{0},+\infty\right)\right), \\
& K_{k}=K \cap\left(\left[x-r_{k-1}, x-r_{k}\right] \cup\left[x+r_{k}, x+r_{k-1}\right]\right), k \in \omega \backslash\{0\} .
\end{aligned}
$$

We see that for all $k \in \omega, x_{k} \in K_{k}$. In addition, the sequence of sets $\left(K_{k}\right)_{k \in \omega}$ satisfies the following properties:

- $K_{k} \subset K$, for all $k \in \omega$.

- $K_{k} \in \mathcal{K}$, for all $k \in \omega$, since they are countable closed subsets of $K$.

- $x_{k} \in K_{k}^{\prime} \neq \varnothing$, for all $k \in \omega$, In fact, let $\varepsilon>0$. First, we consider the case $k \in \omega \backslash\{0\}$. We now take $\hat{\varepsilon}:=\min \left\{\varepsilon, x_{k}-x+r_{k-1}, x-r_{k}-x_{k}\right\}>0$. Since $x_{k} \in K^{\prime}$, there exists $z \in\left[\left(x_{k}-\hat{\varepsilon}, x_{k}+\hat{\varepsilon}\right) \backslash\left\{x_{k}\right\}\right] \cap K$. Thus, $z \in\left[\left(x_{k}-\varepsilon, x_{k}+\varepsilon\right) \backslash\left\{x_{k}\right\}\right] \cap K_{k}$. Hence, $x_{k} \in K_{k}^{\prime}$. For the case $k=0$, by taking $\hat{\varepsilon}:=\min \left\{\varepsilon, x-r_{0}-x_{0}\right\}>0$, and proceeding in a similar way as in the previous case, we see that $x_{0} \in K_{0}^{\prime}$.

- $\left(K_{k}\right)_{k \in \omega}$ is a pairwise disjoint sequence in $\mathcal{K}$.

- $\biguplus_{k \in \omega} K_{k} \biguplus\{x\}=K$. The fact that $\biguplus_{k \in \omega} K_{k} \uplus\{x\} \subset K$ follows directly from (3.1). In order to prove the reverse inclusion, we take $z \in K$. If $z=x$, there is nothing else to show. Now, we suppose that $z \neq x$. Since $r_{n} \rightarrow 0$ as $n \rightarrow+\infty$, we can choose the smallest natural number $N \in \omega$ such that $r_{N}<|x-z|$. Then, $z \in K_{N}$.

Moreover, from (3.1), we see that for all $k \in \omega, x \notin K_{k}^{(\alpha)} \subset\{x\}$. Therefore, for all $k \in \omega, K_{k}^{(\alpha)}=\varnothing$. Thus, for all $k \in \omega, \mathcal{C B}\left(K_{k}\right)=\left(\beta_{k}, p_{k}\right)$ $\in \Omega \times \omega$ implies that $0<\beta_{k}<\alpha$. We remark that for all $k \in \omega, K_{k} \neq \varnothing$ 
implies that $p_{k} \in \omega \backslash\{0\}$. Using the hypothesis, we conclude that for all $k \in \omega$, there exists a homeomorphism $f_{k}$ of $K_{k}$ onto $\omega^{\beta_{k}} \cdot p_{k}+1$. We now define the function

$$
\begin{aligned}
& f: K \mapsto \tau+1 \\
& z \mapsto f(z)= \begin{cases}f_{0}(z), & \text { if } z \in K_{0}, \\
\sum_{j=0}^{k-1} \omega^{\beta_{j}} \cdot p_{j}+1+f_{k}(z), & \text { if } z \in K_{k}, k \in \omega \backslash\{0\}, \\
\tau, & \text { if } z=x,\end{cases}
\end{aligned}
$$

where

$$
\tau:=\sum_{k \in \omega} \omega^{\beta_{k}} \cdot p_{k}:=\sup \left\{\sum_{k=0}^{n} \omega^{\beta_{k}} \cdot p_{k}: n \in \omega\right\}
$$

(a) First, we remark that $f$ is an injective function. In fact, let $u, v \in K$ be such that $f(u)=f(v)$. If $u=x$ and $v \in K_{q}$, for some $q \in \omega$, then $f(v) \leq \sum_{k=0}^{q} \omega^{\beta_{k}} \cdot p_{k}<\tau=f(u)$, which is a contradiction. Thus, there exists $r \in \omega$ such that $u \in K_{r}$. We suppose, by contradiction, that $q \neq r$. Without loss of generality, we may assume that $q<r$. Then,

$$
\begin{aligned}
f(v) & \leq \sum_{k=0}^{n} \omega^{\beta_{k}} \cdot p_{k} \leq \sum_{k=0}^{r-1} \omega^{\beta_{k}} \cdot p_{k} \\
& <\sum_{k=0}^{r-1} \omega^{\beta_{k}} \cdot p_{k}+1+f_{r}(u)=f(u),
\end{aligned}
$$

which is not possible. Hence, $q=r$. Thus,

$$
\sum_{k=0}^{q-1} \omega^{\beta_{k}} \cdot p_{k}+1+f_{q}(u)=f(u)=f(v)=\sum_{k=0}^{q-1} \omega^{\beta_{k}} \cdot p_{k}+1+f_{q}(v),
$$

implies that $f_{q}(u)=f_{q}(v)$. Using the fact that $f_{q}$ is an injective function, it follows that $u=v$. 
(b) We will now show that $f$ is onto. In fact, let $\gamma \leq \tau$. If $\gamma=\tau$, we have that $f(x)=\tau=\gamma$. If $\gamma<\tau$, we take $M:=\min \left\{n \in \omega: \gamma \leq \sum_{k=0}^{n}\right.$ $\left.\omega^{\beta_{k}} \cdot p_{k}\right\}$. In case $M=0, \gamma \leq \omega^{\beta_{0}} \cdot p_{0}$. Since, $f_{0}$ is onto, there exists $z \in K_{0} \subset K$ such that $f(z)=f_{0}(z)=\gamma$. We now assume that $M \in \omega \backslash\{0\}$. Then,

$$
\sum_{k=0}^{M-1} \omega^{\beta_{k}} \cdot p_{k}+1 \leq \gamma \leq \sum_{k=0}^{M} \omega^{\beta_{k}} \cdot p_{k} .
$$

Thus, there exists an ordinal number $\mu$ such that

$$
\sum_{k=0}^{M-1} \omega^{\beta_{k}} \cdot p_{k}+1+\mu=\gamma \leq \sum_{k=0}^{M-1} \omega^{\beta_{k}} \cdot p_{k}+\omega^{\beta_{M}} \cdot p_{M}
$$

Then, $\mu \leq \omega^{\beta_{M}} \cdot p_{M}$. Since $f_{M}$ is onto, there exists $z \in K_{M} \subset K$ such that $f_{M}(z)=\mu$. So, $f(z)=\sum_{k=0}^{M-1} \omega^{\beta_{k}} \cdot p_{k}+1+f_{M}(z)=\gamma$.

(c) Moreover, for all $k \in \omega,\left.f\right|_{K_{k}}$ equals an ordinal number, i.e., a constant function, plus a continuous function. Thus, for all $k \in \omega,\left.f\right|_{K_{k}}$ is a continuous function. In addition, since $\left(K_{k}\right)_{k \in \omega}$ is a pairwise disjoint sequence of open subsets in $K$, it follows that $f$ is a continuous function at any element of $\biguplus_{k \in \omega} K_{k}$. Furthermore, $f$ is also continuous at the point $x \in K$. If fact, let $\mu$ be an ordinal number such that $\mu<\tau$. There exists $m \in \omega$ such that $\mu<\sum_{j=0}^{m} \omega^{\beta_{j}} \cdot p_{j}$. We claim that

$$
f\left(\left(x-r_{m}, x+r_{m}\right) \cap K\right) \subset(\mu, \tau+1) .
$$

Let $y \in\left(x-r_{m}, x+r_{m}\right) \cap K$. If $y=x$, then $f(y)=f(x)=\tau \in(\mu, \tau+1)$. We now suppose that $y \neq x$. Then, there is $i \in \omega$ such that $y \in K_{i}$. Since $\left(r_{n}\right)_{n \in \omega}$ is a strictly decreasing sequence of positive numbers, we conclude that $i>m$. Then, 


$$
f(y)=\sum_{j=0}^{i-1} \omega^{\beta_{j}} \cdot p_{j}+1+f_{i}(y) \geq \sum_{j=0}^{m} \omega^{\beta_{j}} \cdot p_{j}>\mu .
$$

Moreover,

$$
\begin{aligned}
f(y) & =\sum_{j=0}^{i-1} \omega^{\beta_{j}} \cdot p_{j}+1+f_{i}(y) \leq \sum_{j=0}^{i-1} \omega^{\beta_{j}} \cdot p_{j}+1+\omega^{\beta_{i}} \cdot p_{i} \\
& =\sum_{j=0}^{i} \omega^{\beta_{j}} \cdot p_{j} \leq \tau<\tau+1 .
\end{aligned}
$$

From Equations (3.3) and (3.4), we see that $f(y) \in(\mu, \tau+1)$. Thus, Equation (3.2) follows. Hence, $f$ is continuous at the point $x$.

By (a) and (b), $f$ is a bijective function. In addition, by (c), $f$ is a continuous function of $K$ onto $\tau+1$.

We will now prove that $\tau=\omega^{\alpha}$. In order to get this, let $\tilde{\alpha}:=\sup \left\{\beta_{k}\right.$ : $k \in \omega\} \in \mathbf{O R}$. We see that $\tilde{\alpha} \leq \alpha$.

(i) First, we consider the case $\tilde{\alpha}<\alpha$. Then, $\tilde{\alpha}+1 \leq \alpha$. Thus, for all $k \in \omega, K_{k}^{(\widetilde{\alpha}+1)}=\varnothing$. Using transfinite induction, and proceeding as in the proof of (2.2), we get

$$
K^{(\widetilde{\alpha}+1)}=\biguplus_{k \in \omega} K_{k}^{(\widetilde{\alpha}+1)} \biguplus\{x\}=\{x\} .
$$

Then, $\widetilde{\alpha}+1=\alpha$. Since for all $k \in \omega, \omega^{\beta_{k}} \cdot p_{k} \leq \omega^{\tilde{\alpha}} \cdot p_{k}$, we see that

$$
\tau=\sum_{k \in \omega} \omega^{\beta_{k}} \cdot p_{k} \leq \omega^{\tilde{\alpha}} \cdot\left(\sum_{k \in \omega} p_{k}\right)=\omega^{\tilde{\alpha}} \cdot \omega=\omega^{\tilde{\alpha}+1}=\omega^{\alpha} .
$$

On the other hand, we claim that

$$
\left|\left\{n \in \omega: \beta_{n}=\tilde{\alpha}\right\}\right|=\aleph_{0} .
$$

In order to prove (3.6), we first suppose, by contradiction, that for all $n \in \omega, \beta_{n}<\tilde{\alpha}$. Thus, for all $n \in \omega, \beta_{n}+1 \leq \widetilde{\alpha}$, and we get $K_{n}^{(\widetilde{\alpha})} \subset$ 
$K_{n}^{\left(\beta_{n}+1\right)}=\varnothing$. Moreover, we see that $K^{(\widetilde{\alpha})}=\biguplus_{k \in \omega} K_{k}^{(\widetilde{\alpha})} \biguplus\{x\}=\{x\}$. Then, $\widetilde{\alpha}=\alpha$, which is a contradiction. Hence, there exists at least one $n \in \omega$ such that $\beta_{n}=\widetilde{\alpha}$. We now suppose, again by contradiction, that the set $\left\{n \in \omega: \beta_{n}=\tilde{\alpha}\right\} \neq \varnothing$ is finite. Let $N:=\max \left\{n \in \omega: \beta_{n}=\tilde{\alpha}\right\} \in \omega$. We have that for all $k \in \omega$ such that $k>N, \beta_{k}<\tilde{\alpha}$. Then,

$$
K^{(\widetilde{\alpha})}=\biguplus_{k \in \omega} K_{k}^{(\widetilde{\alpha})} \biguplus\{x\}=\biguplus_{k=0}^{N} K_{k}^{(\widetilde{\alpha})} \biguplus\{x\} .
$$

It follows that, $K^{(\widetilde{\alpha})}$ is a finite set. Hence, $K^{(\alpha)}=K^{(\tilde{\alpha}+1)}=\varnothing$, which is a contradiction with the fact that $K^{(\alpha)}=\{x\}$. Therefore, (3.6) is proved. We now define, for all $n \in \omega$,

$$
m_{n}:=\mid\left\{k \in \omega: k \leq n \text { and } \beta_{k}=\tilde{\alpha}\right\} \mid \in \omega .
$$

Then, for all $n \in \omega$, we have that

$$
\sum_{k=0}^{n} \omega^{\beta_{k}} \cdot p_{k} \geq \omega^{\widetilde{\alpha}} \cdot m_{n} .
$$

For this reason,

$$
\begin{aligned}
\tau & =\sum_{k \in \omega} \omega^{\beta_{k}} \cdot p_{k} \geq \omega^{\tilde{\alpha}} \cdot \sup \left\{m_{n}: n \in \omega\right\} \\
& =\omega^{\tilde{\alpha}} \cdot \omega=\omega^{\tilde{\alpha}+1}=\omega^{\alpha} .
\end{aligned}
$$

Using (3.5) and (3.7), we conclude that $\tau=\omega^{\alpha}$.

(ii) We now consider the case $\tilde{\alpha}=\alpha$. We claim that for all $k \in \omega$, $\beta_{k}<\tilde{\alpha}$. In fact, if there exists $l \in \omega$ such that $\beta_{l}=\tilde{\alpha}$, then

$$
K_{l}^{\left(\beta_{l}\right)} \biguplus\{x\} \subset \biguplus_{i \in \omega} K_{i}^{\left(\beta_{l}\right)} \biguplus\{x\}=K^{\left(\beta_{l}\right)}=K^{(\alpha)}=\{x\},
$$

contradicting the fact that $\left|K_{l}^{\left(\beta_{l}\right)}\right|=p_{l}>0$. We now remark that $\alpha$ is a limit ordinal. In order to prove the last assertion, we suppose, for the 
sake of contradiction, that $\alpha$ is a successor ordinal. Then, there exists an ordinal number $\lambda$ such that $\alpha=\lambda+1$. Thus, for all $k \in \omega, \beta_{k} \leq \lambda<\alpha=\widetilde{\alpha}$, which is a contradiction with the definition of $\tilde{\alpha}$. On the other hand, since for all $k \in \omega, \omega^{\beta_{k}} \leq \omega^{\beta_{k}} \cdot p_{k} \leq \tau$, it follows that

$$
\omega^{\alpha}=\omega^{\widetilde{\alpha}}=\sup \left\{\omega^{\beta_{k}}: k \in \omega\right\} \leq \tau .
$$

We now define, for all $n \in \omega$,

$$
\begin{aligned}
\beta_{k_{n}} & :=\max \left\{\beta_{k}: k=0,1, \ldots, n\right\}, \\
p_{k_{n}} & :=\max \left\{p_{k}: k=0,1, \ldots, n\right\} .
\end{aligned}
$$

Then, for all $n \in \omega$, we see that

$$
\sum_{k=0}^{n} \omega^{\beta_{k}} \cdot p_{k} \leq \omega^{\beta_{k_{n}}} \cdot p_{k_{n}} \cdot n<\omega^{\beta_{k_{n}+1}} \leq \omega^{\alpha},
$$

where in the last inequality we have used the fact that $\beta_{k_{n}}<\beta_{k_{n}}+1 \leq \alpha$. In consequence,

$$
\tau=\sum_{k \in \omega} \omega^{\beta_{k}} \cdot p_{k} \leq \omega^{\alpha}
$$

Equations (3.8) and (3.9) imply that $\tau=\omega^{\alpha}$.

Therefore, $f$ is a bijective and continuous function of $K$ onto $\tau+1=\omega^{\alpha}+1$. In addition, since $\omega^{\alpha}+1$ is a Hausdorff space, we conclude that $f$ is a homeomorphism of $K$ onto $\omega^{\alpha}+1$.

Lemma 3.4. Suppose that $K$ and $F$ are closed subsets of $\mathbb{R}$ such that $K \cap F=K \cap \stackrel{\circ}{F}$, where $\stackrel{\circ}{F}$ is the set of all interior points of $F$. Then, for all $\alpha \in \mathbf{O R}$, we have that

$$
(K \cap F)^{(\alpha)}=K^{(\alpha)} \cap F .
$$


Proof. We proceed by transfinite induction.

- The case $\alpha=0$ is immediate.

- We now suppose that the result is true for $\alpha \in \mathbf{O R}$. Then,

$(K \cap F)^{(\alpha+1)}=\left((K \cap F)^{(\alpha)}\right)^{\prime}=\left(K^{(\alpha)} \cap F\right)^{\prime} \subset\left(K^{(\alpha)}\right)^{\prime} \cap F^{\prime} \subset K^{(\alpha+1)} \cap F$,

where in the last expression we have used the induction hypothesis and the fact that $F$ is closed. In order to prove the reverse inclusion, let $x \in K^{(\alpha+1)} \cap F$. Since $K$ is closed, $x \in K \cap F=K \cap \stackrel{\circ}{F}$. Thus, there exists $r>0$ such that $(x-r, x+r) \subset F$. Let $\varepsilon>0$. We now take $\widetilde{\varepsilon}:=\min \{\varepsilon, r\}>0$. Then,

$$
\begin{aligned}
\varnothing & \neq((x-\widetilde{\varepsilon}, x+\widetilde{\varepsilon}) \backslash\{x\}) \cap K^{(\alpha)}=((x-\widetilde{\varepsilon}, x+\widetilde{\varepsilon}) \backslash\{x\}) \cap K^{(\alpha)} \cap F \\
& \subset((x-\varepsilon, x+\varepsilon) \backslash\{x\}) \cap(K \cap F)^{(\alpha)} .
\end{aligned}
$$

Hence, $x \in(K \cap F)^{(\alpha+1)}$. Therefore, $(K \cap F)^{(\alpha+1)}=K^{(\alpha+1)} \cap F$.

- Finally, let $\lambda \neq 0$ be a limit ordinal number. We suppose that for all $\beta \in \mathbf{O R}$ such that $\beta<\lambda,(K \cap F)^{(\beta)}=K^{(\beta)} \cap F$. Then,

$$
(K \cap F)^{(\lambda)}=\bigcap_{\beta<\lambda}(K \cap F)^{(\beta)}=\bigcap_{\beta<\lambda}\left(K^{(\beta)} \cap F\right)=\bigcap_{\beta<\lambda} K^{(\beta)} \cap F=K^{(\lambda)} \cap F .
$$

This concludes the proof.

Lemma 3.5. Let $\alpha$ be a countable ordinal number such that $\alpha>0$. Let $p \in \omega \backslash\{0\}$. Suppose that for all $\tilde{K} \in \mathcal{K}$ such that $\mathcal{C B}(\widetilde{K})=(\alpha, 1)$, there exists a homeomorphism of $\widetilde{K}$ onto $\omega^{\alpha}+1$. Then, for all $K \in \mathcal{K}$ such that $\mathcal{C B}(K)=(\alpha, p)$, there exists a homeomorphism of $K$ onto $\omega^{\alpha} \cdot p+1$. 
Proof. Let $K \in \mathcal{K}$ be such that $\mathcal{C B}(K)=(\alpha, p) \in \Omega \times \omega$. We write $K^{(\alpha)}=\left\{x_{1}, x_{2}, \ldots, x_{p}\right\}$, where $x_{i}<x_{j}$, for all $i, j \in I:=\{1, \ldots, p\}$ with $i<j$. We see that for all $k \in\{1, \ldots, p-1\}$, there exists $z_{k} \in\left(x_{k}, x_{k+1}\right)$ such that $z_{k} \notin K$. We now consider the sets

$$
\begin{aligned}
K_{1} & =K \cap\left(-\infty, z_{1}\right], \\
K_{k} & =K \cap\left[z_{k-1}, z_{k}\right], k \in\{2, \ldots, p-1\}, \\
K_{p} & =K \cap\left[z_{p-1},+\infty\right) .
\end{aligned}
$$

Proceeding as in the proof of Lemma 3.3, it is possible to show that the finite family $\left(K_{k}\right)_{k \in I}$ satisfies the following properties:

- $K_{k} \subset K$, for all $k \in I$.

- $K_{k} \in \mathcal{K}$, for all $k \in I$.

- $x_{k} \in K_{k}^{\prime} \neq \varnothing$, for all $k \in I$.

- $\left(K_{k}\right)_{k \in I}$ is a pairwise disjoint finite sequence in $\mathcal{K}$.

- $\biguplus_{k \in I} K_{k}=K$.

By using Lemma 3.4, we have that for all $k \in I, K_{k}^{(\alpha)}=\left\{x_{k}\right\}$. Therefore, for all $k \in I, \mathcal{C B}\left(K_{k}\right)=(\alpha, 1)$. Thus, for all $k \in I$, there exists a homeomorphism $f_{k}$ of $K_{k}$ onto $\omega^{\alpha}+1$. We now define the function $f$ given by

$$
\begin{aligned}
f: K \mapsto \tau+1 & \\
z & \mapsto f(z)= \begin{cases}f_{1}(z), & \text { if } z \in K_{1}, \\
\sum_{j=1}^{k-1} \omega^{\alpha}+1+f_{k}(z), & \text { if } z \in K_{k}, \text { for some } k \in I \backslash\{1\},\end{cases}
\end{aligned}
$$


where

$$
\tau:=\sum_{j=1}^{p} \omega^{\alpha}=\omega^{\alpha} \cdot \sum_{j=1}^{p} 1=\omega^{\alpha} \cdot p .
$$

Proceeding in a similar fashion as in the items (a), (b), and (c) in the proof of Lemma 3.3, we obtain that $f$ is a homeomorphism of $K$ onto $\omega^{\alpha} \cdot p+1$.

Lemma 3.6. Let $\alpha$ be a countable ordinal number such that $\alpha>0$. Let $p \in \omega \backslash\{0\}$. Then, for all $K \in \mathcal{K}$ such that $\mathcal{C B}(K)=(\alpha, p)$, there exists a homeomorphism of $K$ onto $\omega^{\alpha} \cdot p+1$.

Proof. We will use strong transfinite induction. By Lemmas 3.2 and 3.5, the result holds for $\alpha=1$. We now consider $\alpha \in \Omega$ such that $\alpha>1$, and we suppose that the result is true for all ordinal number $\beta$ such that $0<\beta<\alpha$. Lemmas 3.3 and 3.5 imply the result for $\alpha$. Hence, the lemma is proved.

Next result contains the reciprocal of Theorem 3.2.

Theorem 3.3. If $K_{1}, K_{2} \in \mathcal{K}$ and $\mathcal{C B}\left(K_{1}\right)=\mathcal{C B}\left(K_{2}\right)$, then $K_{1} \sim K_{2}$.

Proof. If $\mathcal{C B}\left(K_{1}\right)=\mathcal{C B}\left(K_{2}\right)=(0, p) \in \Omega \times \omega$, we get $\left|K_{1}\right|=\left|K_{2}\right|=p$. Then, $K_{1} \sim K_{2}$.

We now suppose that $\mathcal{C B}\left(K_{1}\right)=\mathcal{C B}\left(K_{2}\right)=(\alpha, p)$, with $\alpha>0$. By Proposition 3.1, $p \in \omega \backslash\{0\}$. By Lemma 3.6, there exist two homeomorphisms, $g$ of $K_{1}$ onto $\omega^{\alpha} \cdot p+1$ and $h$ of $K_{2}$ onto $\omega^{\alpha} \cdot p+1$. Therefore, $f=h^{-1} \circ g: K_{1} \mapsto K_{2}$ is a homeomorphism of $K_{1}$ onto $K_{2}$. Hence, $K_{1} \sim K_{2}$.

Theorems 3.2 and 3.3 fully characterize the partition of $\mathcal{K}$ by the Cantor-Bendixson characteristic. 


\subsection{Cardinality of the set $\mathscr{K}$}

Combining the previous results we obtain the cardinality of $\mathscr{K}$.

Theorem 3.4. The set $\mathscr{K}$, given by (1. 4), has cardinality $\aleph_{1}$.

Proof. We define the function

$$
\begin{aligned}
\widetilde{\mathcal{C B}}: \mathscr{K} & \mapsto(\Omega \times(\omega \backslash\{0\})) \cup(0,0) \\
{[K] } & \mapsto \widetilde{\mathcal{C B}}([K])=\mathcal{C B}(K)=(\alpha, p) .
\end{aligned}
$$

By Theorem 3.2 and Proposition 3.1, we see that $\widetilde{\mathcal{C B}}$ is well-defined. Moreover, Corollary 2.1 implies that $\widetilde{\mathcal{C B}}$ is a surjective function. Furthermore, by Theorem 3.3, $\widetilde{\mathcal{C B}}$ is an injective function. Then,

$$
|\mathscr{K}|=|(\Omega \times(\omega \backslash\{0\})) \cup(0,0)|=|\Omega \times \omega|=|\Omega|=\aleph_{1} .
$$

Last theorem shows that

$$
\aleph_{0}<\aleph_{1}=|\mathscr{K}| \leq 2^{\aleph_{0}}=\mathfrak{c}
$$

where $\mathfrak{c}$ is the cardinality of $\mathbb{R}$.

\subsection{A "primitive" related to the Cantor-Bendixson derivative of compact subsets of the real line}

We end this paper with a last theorem that we can view as a generalization of Theorem 2.1 and Corollary 2.1 given in Section 2. The next result shows that for any compact subset of the reals, there is a primitive-like set associated to its Cantor-Bendixson derivative.

Theorem 3.5. Suppose that $\alpha \in \Omega$. Let $F$ be a compact subset of $\mathbb{R}$. Then, there exists a compact set $\mathcal{F} \subset \mathbb{R}$ such that $\mathcal{F}^{(\alpha)}=F$.

Proof. If $\alpha=0$, we define $\mathcal{F}=F$ and the result holds.

From now on, we suppose that $\alpha>0$. There are two cases. First, if $F$ is perfect, i.e., $F=F^{\prime}$, we can take $\mathcal{F}=F$, and the result follows. 
We now assume that $F \neq F^{\prime}$. Since $F \backslash F^{\prime}$ is the set of all isolated points of $F$, we have that $F \backslash F^{\prime} \neq \varnothing$ is countable. Hence, $F \backslash F^{\prime}=$ $\left\{x_{n}: n \in I\right\}$, where $\varnothing \neq I \subset \omega$, and $x_{n} \neq x_{m}$, for all $n, m \in I$ with $n \neq m$. Furthermore, for all $n \in I$, there exists $r_{n} \in\left(0, \frac{1}{n+1}\right)$ such that $\left(x_{n}-r_{n}, x_{n}+r_{n}\right) \cap F=\left\{x_{n}\right\}$. By Theorem 2.1, we see that for all $n \in I$, there exits $K_{n} \in \mathcal{K}$ such that $K_{n} \subset\left(x_{n}-r_{n}, x_{n}\right]$ and $K_{n}^{(\alpha)}=\left\{x_{n}\right\}$. Since $\left(\left(x_{n}-r_{n}, x_{n}\right]\right)_{n \in I}$ is a pairwise disjoint sequence of intervals, we see that $\left(K_{n}\right)_{n \in I}$ is a pairwise disjoint sequence in $\mathcal{K}$. We now define the set $\mathcal{F} \subset \mathbb{R}$ given by

$$
\mathcal{F}:=\biguplus_{n \in I} K_{n} \cup F
$$

Claim 1. $\mathcal{F}$ is a compact subset of $\mathbb{R}$.

In fact, let $\left(z_{k}\right)_{k \in \omega}$ be a sequence in $\mathcal{F}$ such that $z_{k} \rightarrow z \in \mathbb{R}$ when $k \rightarrow+\infty$. There are three cases.

(i) If $\left\{k \in \omega: z_{k} \in F\right\}$ is infinite, there exists a subsequence $\left(z_{\phi(k)}\right)_{k \in \omega}$ in $F$, where $\phi: \omega \mapsto \omega$ is a strictly increasing function. Since $F$ is closed, we conclude that $z \in F \subset \mathcal{F}$.

(ii) We now suppose that there exists $m \in I$ such that $\left\{k \in \omega: z_{k} \in K_{m}\right\}$ is infinite. Similarly as in the previous case, we obtain that $z \in K_{m} \subset \mathcal{F}$.

(iii) Finally, we assume that for all $n \in I,\left\{k \in \omega: z_{k} \in K_{n}\right\}$ is a finite set and $\left\{k \in \omega: z_{k} \in F\right\}$ is also finite. Thus, there exists a subsequence $\left(z_{\psi(k)}\right)_{k \in \omega}$, where $\quad \psi: \omega \mapsto \omega$ is a strictly increasing function, and there is also a strictly increasing function $\sigma: \omega \mapsto I$ such that for all $k \in \omega$

$$
z_{\psi(k)} \in K_{\sigma(k)} \subset\left(x_{\sigma(k)}-r_{\sigma(k)}, x_{\sigma(k)}\right] .
$$


In order to prove the last assertion, we see that there exists $n_{0} \in I$ such that $\left\{k \in \omega: z_{k} \in K_{n_{0}}\right\} \neq \varnothing$. Then, there is $k_{0} \in \omega$ with $z_{k_{0}} \in K_{n_{0}}$. We thus define $\psi(0):=k_{0}$ and $\sigma(0):=n_{0}$. We now get $n_{1} \in I$ with $n_{1}>n_{0}$ and such that $\left\{k \in \omega: z_{k} \in K_{n_{1}}, k>k_{0}\right\} \neq \varnothing$. So, there exists $k_{1} \in \omega$ with $k_{1}>k_{0}$ and such that $z_{k_{1}} \in K_{n_{1}}$. We define $\psi(1):=k_{1}$ and $\sigma(1):=n_{1}$. By continuing this process, functions $\psi$ and $\sigma$ are recursively obtained. From (3.14), we have that for all $k \in \omega,\left|x_{\sigma(k)}-z_{\psi(k)}\right|<r_{\sigma(k)}<$ $\frac{1}{\sigma(k)+1}$. As $\left(z_{\psi(k)}\right)_{k \in \omega}$ converges to $z$, it follows that $\left(x_{\sigma(k)}\right)_{k \in \omega}$ also converges to $z$. Since, the elements of the last sequence belong to $F$, and $F$ is closed, we conclude that $z \in F \subset \mathcal{F}$.

From (i), (ii), and (iii), $\mathcal{F}$ is a closed subset of $\mathbb{R}$. Moreover, since $F$ is bounded, there exist $a, b \in \mathbb{R}$, with $a<b$, such that $F \subset[a, b]$. Then, $\mathcal{F} \subset[a-1, b]$, i.e., $\mathcal{F}$ is bounded. Hence, $\mathcal{F}$ is a compact subset of $\mathbb{R}$.

Claim 2. $\mathcal{F}^{(\alpha)}=F$.

Actually, we will show the following more general result: For all countable ordinal number $\beta \in \Omega$ such that $\beta \leq \alpha$

$$
\mathcal{F}^{(\beta)}=\biguplus_{n \in I} K_{n}^{(\beta)} \cup F
$$

In order to prove (3.15), we proceed by transfinite induction as in Theorem 2.1.

(a) If $\beta=0$, then the result holds immediately.

(b) We now suppose that (3.15) is true for a given $\beta \in \Omega$ such that $\beta<\alpha$. We note that for all $n \in I, K_{n}^{(\beta+1)} \subset \mathcal{F}^{(\beta+1)}$. Then,

$$
\biguplus_{n \in I} K_{n}^{(\beta+1)} \subset \mathcal{F}^{(\beta+1)} .
$$

Furthermore, by the induction hypothesis, $F \subset \mathcal{F}^{(\beta)}$. Then, $F^{\prime} \subset \mathcal{F}^{(\beta+1)}$. Moreover, 


$$
F \backslash F^{\prime}=\biguplus_{n \in I}\left\{x_{n}\right\}=\biguplus_{n \in I} K_{n}^{(\alpha)} \subset \biguplus_{n \in I} K_{n}^{(\beta+1)} \subset \mathcal{F}^{(\beta+1)} .
$$

Hence,

$$
\biguplus_{n \in I} K_{n}^{(\beta+1)} \cup F \subset \mathcal{F}^{(\beta+1)} .
$$

In order to show the reverse inclusion, we take $x \in \mathcal{F}^{(\beta+1)}$. Using the induction hypothesis, we see that

$$
x \in \mathcal{F}^{(\beta+1)}=\left(\mathcal{F}^{(\beta)}\right)^{\prime}=\left(\biguplus_{n \in I} K_{n}^{(\beta)} \cup F\right)^{\prime}=\left(\biguplus_{n \in I} K_{n}^{(\beta)}\right)^{\prime} \cup F^{\prime} .
$$

Using now Claim 1, we have that $\mathcal{F}$ is closed. Then,

$$
x \in \mathcal{F}^{(\beta+1)} \subset \mathcal{F}^{(\beta)}=\biguplus_{n \in I} K_{n}^{(\beta)} \cup F .
$$

If $x \in F$, there is nothing left to show. On the other hand, if $x \notin F$, there exists $m \in I$ such that $x \in K_{m}^{(\beta)} \subset\left(x_{m}-r_{m}, x_{m}\right]$. We now assume, by contradiction, that $x \notin K_{m}^{(\beta+1)}$. Then, $x$ is an isolated point of $K_{m}^{(\beta)}$.

Since $x \neq x_{m} \in F$, there is $0<\varepsilon<\min \left\{x-x_{m}+r_{m}, x_{m}-x\right\}$ such that

$$
(x-\varepsilon, x+\varepsilon) \cap K_{m}^{(\beta)}=\{x\} .
$$

Moreover, as $(x-\varepsilon, x+\varepsilon) \subset\left(x_{m}-r_{m}, x_{m}\right)$, we conclude that for all $n \in I$ with $n \neq m$,

$$
(x-\varepsilon, x+\varepsilon) \cap K_{n}^{(\beta)}=\varnothing .
$$

Then,

$$
(x-\varepsilon, x+\varepsilon) \cap \biguplus_{n \in I} K_{n}^{(\beta)}=\{x\} .
$$


Therefore, $x$ is an isolated point of $\biguplus_{n \in I} K_{n}^{(\beta)}$. Since $x \notin F$, and $F$ is closed, we see that $x \notin F^{\prime}$. Hence, $x \in\left(\biguplus_{n \in I} K_{n}^{(\beta)}\right)^{\prime}$, which is contradictory. In consequence,

$$
x \in K_{m}^{(\beta+1)} \subset \biguplus_{n \in I} K_{n}^{(\beta+1)} .
$$

Thus, summarizing, we can conclude that

$$
\mathcal{F}^{(\beta+1)} \subset \biguplus_{n \in I} K_{n}^{(\beta+1)} \cup F .
$$

From (3.16) and (3.17), we get

$$
\mathcal{F}^{(\beta+1)}=\biguplus_{n \in I} K_{n}^{(\beta+1)} \cup F .
$$

(c) Finally, let $\gamma \neq 0$ be a limit ordinal such that $\gamma \leq \alpha$ and we assume that for all ordinal number $\delta$ such that $\delta<\gamma$,

$$
\mathcal{F}^{(\delta)}=\biguplus_{n \in I} K_{n}^{(\delta)} \cup F
$$

Using (3.18), we obtain

$$
\begin{aligned}
\biguplus_{n \in I} K_{n}^{(\gamma)} \cup F & =\biguplus_{n \in I}\left(\bigcap_{\delta<\gamma} K_{n}^{(\delta)}\right) \cup F \\
& \subset \bigcap_{\delta<\gamma}\left(\biguplus_{n \in I} K_{n}^{(\delta)}\right) \cup F \\
& =\bigcap_{\delta<\gamma}\left(\biguplus_{n \in I} K_{n}^{(\delta)} \cup F\right) \\
& =\bigcap_{\delta<\gamma} \mathcal{F}^{(\delta)} \\
& =\mathcal{F}^{(\gamma)} .
\end{aligned}
$$


In order to show the other inclusion, we take $x \in \mathcal{F}^{(\gamma)}$. Using the induction hypothesis (3.18), we see that

$$
\mathcal{F}^{(\gamma)}=\bigcap_{\delta<\gamma} \mathcal{F}^{(\delta)}=\bigcap_{\delta<\gamma}\left(\biguplus_{n \in I} K_{n}^{(\delta)} \cup F\right) .
$$

Then, either $x \in F$ or for all ordinal number $\delta$ such that $\delta<\gamma$, there exists $n \in I$ such that $x \in K_{n}^{(\delta)}$. If $x \in F$, then there is nothing else to be done. If $x \notin F$, there is $N \in I$ such that $x \in K_{N}^{(0)}=K_{N}$. We now assume, to get a contradiction, that there is an ordinal number $\delta_{0}$ with $\delta_{0}<\gamma$ and such that $x \notin K_{N}^{\left(\delta_{0}\right)}$. Since there is $l \in I$ with $l \neq N$ such that $x \in K_{l}^{\left(\delta_{0}\right)} \subset K_{l}$, we obtain a contradiction with the fact that $K_{l} \cap K_{N}=\varnothing$. Hence, for all ordinal number $\delta$ such that $\delta<\gamma, x \in K_{N}^{(\delta)}$. In consequence,

$$
x \in \bigcap_{\delta<\gamma} K_{N}^{(\delta)}=K_{N}^{(\gamma)} \subset \biguplus_{n \in I} K_{n}^{(\gamma)} .
$$

Thus,

$$
\mathcal{F}^{(\gamma)} \subset \biguplus_{n \in I} K_{n}^{(\gamma)} \cup F
$$

From (3.19) and (3.20), we have that

$$
\mathcal{F}^{(\gamma)}=\biguplus_{n \in I} K_{n}^{(\gamma)} \cup F .
$$

By (a), (b) and (c), we obtain (3.15) for all countable ordinal number $\beta$ such that $\beta \leq \alpha$. Finally, using (3.15) with $\alpha$, we get

$$
\mathcal{F}^{(\alpha)}=\biguplus_{n \in I} K_{n}^{(\alpha)} \cup F=\biguplus_{n \in I}\left\{x_{n}\right\} \cup F=F,
$$

which finishes the proof. 


\section{References}

[1] G. Cantor, Ueber die Ausdehnung eines Satzes aus der Theorie der trigonometrischen Reihen, Math. Ann. 5 (1872), 123-132.

[2] G. Cantor, Ueber unendliche, lineare Punktmannichfaltigkeiten I, Math. Ann. 15 (1879), 1-7.

[3] G. Cantor, Ueber unendliche, lineare Punktmannichfaltigkeiten II, Math. Ann. 17 (1880), 355-358.

[4] G. Cantor, Ueber unendliche, lineare Punktmannichfaltigkeiten III, Math. Ann. 20 (1882), 113-121.

[5] G. Cantor, Sur divers théorèmes de la théorie des ensembles de points situés dans un espace continu à $\mathrm{n}$ dimensions, Acta Math. 2 (1883), 409-414.

[6] G. Cantor, Ueber unendliche, lineare Punktmannichfaltigkeiten IV, Math. Ann. 21 (1883), 51-58.

[7] S. Mazurkiewicz and W. Sierpinski, Contribution à la topologie des ensembles dénombrables, Fund. Math. 1 (1920), 17-27. 